\title{
Building the second version of the World Digital Magnetic Anomaly Map (WDMAM)

\author{
Vincent Lesur $^{{ }^{*}}$ (D), Mohamed Hamoudi ${ }^{2,3}$, Yujin Choi $^{1}$, Jérôme Dyment ${ }^{1}$ and Erwan Thébault ${ }^{4}$
}

\begin{abstract}
The World Digital Anomaly Map (WDMAM) is a worldwide compilation of near-surface magnetic data. We present here a candidate for the second version of the WDMAM and its characteristics. This candidate has been evaluated by a group of independent reviewers and has been adopted as the official second version of the WDMAM during the 26th general assembly of the International Union of Geodesy and Geomagnetism (IUGG). The way this compilation has been built is described with some details. A global magnetic field model of the lithosphere contribution, parameterised by spherical harmonics, has been derived up to degree and order 800 . The model information content has been evaluated by computing local spectra. Further, the compatibility of the anomaly field displayed by the WDMAM with a pure induced magnetisation is tested by comparison with the main field strength. These studies allowed an analysis of the compilation in terms of strength and wavelength content. They confirm the extremely smooth and weak contribution of the magnetic field generated in the lithosphere over Western Europe. This apparent weakness possibly extends to the Northern African continent. However, a global analysis remains difficult to achieve given the sparseness of good quality data over very large area of oceans and continents. The WDMAM and related information can be downloaded at http://www.wdmam.org/.
\end{abstract}

Keywords: Geomagnetism, Lithosphere, Anomaly field, Magnetic field model

\section{Introduction}

The World Digital Magnetic Anomaly Map (WDMAM) task force is part of the working group V-mod of the International Association of Geomagnetism and Aeronomy (IAGA). It was formed in 2003 and aims at collecting aeromagnetic and marine data worldwide to provide the scientific community with a compilation of these data on a global, $5-\mathrm{km}$ cell size, grid. The first version of the WDMAM was released in 2007 (Korhonen et al. 2007). Several groups proposed candidates for this first version of the map (e.g. Hamoudi et al. 2007; Maus et al. 2007), not all associated with a publication, but each with its own way of processing and merging the same data set. None of the candidates was significantly better than the others, and the final map was built by merging and reprocessing several of the candidates. Following this work, Maus et al. continued collecting data and proposed their own

${ }^{*}$ Correspondence: lesur@ipgp.fr

1 Institut de Physique du Globe de Paris, CNRS UMR 7154, 1 rue Jussieu, 75005 Paris, France

Full list of author information is available at the end of the article map and associated magnetic field model covering spherical harmonic (SH) degrees from 16 to 720 (Maus et al. 2009; Maus 2010).

No data is included in the WDMAM without prior agreement of the data owner. This clearly slows down the data collection process and only a few new data sets have been made available since the release of the first grid. However, the main limitation of this grid was the handling of marine data and particularly the way the oceanic data gaps were filled. This triggered the decision to produce a second version of the map. In this work, we present a candidate for the second version of the WDMAM. It has been built by two groups that were originally working independently in the "Institut de Physique du Globe" in Paris (IPGP) and in the "GeoForschungsZentrum" in Potsdam (GFZ). This candidate is the only candidate that has been proposed; it has nonetheless been evaluated by independent scientists and modified before becoming the official second version of the WDMAM. This version has been approved by IAGA during the 26th IUGG assembly in 2015 and publicly released. It can be dowloaded at www. wdmam.org.

\section{实 Springer}


We derived this version of the map trying to remain as close as possible to the original aeromagnetic data. We therefore have been careful in limiting the overlap between data sets so that each data set can be clearly identified through an index. Only the long wavelengths of a given data set may have been modified, and we provide the grid with enough information so that these long wavelengths can be modified again by the users. Significant efforts have been made to produce better anomaly patterns over the oceans; they are described in the next section, together with the work done on the continental data, and the merging process leading to the final candidate. We also found useful to derive a model of the lithospheric field from this WDMAM candidate. This model goes up to $\mathrm{SH}$ degree and order 800 and is described in the third section. In the last section, we discuss some of the aspects of the grid and its associated model.

\section{Data sets and processing Marine data sets}

Marine magnetic data acquired by a variety of oceanographic institutions and ships worldwide are gathered by the World Data Service (WDS) for Geophysics operated by the National Oceanic and Atmospheric Administration (NOAA), Boulder, CO, USA (see http://www.ngdc. noaa.gov/mgg/geodas/trackline.html). These data have been carefully reprocessed by Quesnel et al. (2009), who performed extensive data cleaning, removal of the core and external fields using the comprehensive model CM4 (Sabaka et al. 2004), and line levelling. The data set produced by these authors (Quesnel et al. 2009) has been the base of the WDMAM version 1 (Korhonen et al. 2007) over the oceans. We use the same data set, complemented by additional marine data made available by NOAA between 2006 and 2010 and processed in the same way (Takemi Ishihara and Manuel Catalan, personal communication, 2011), as the base of the WDMAM version 2 over the oceans. To produce the grid, these data sets have been interpolated using the GMT function nearneighbor with either a $40-\mathrm{km}$ search radius requiring data in each on the four quadrants, or a $5-\mathrm{km}$ search radius requiring data only in one quadrant. This approach leads to a homogeneous grid for area with a high density of data and the preservation of the tracks when these are isolated.

If the data coverage is relatively good in the North and Central Atlantic Ocean as well as in the NorthEastern and North-Western Pacific Ocean, it is particularly sparse in the remote parts of the Pacific, Indian and Southern Oceans. Two methods have been proposed to fill the gaps in these areas. The WDMAM version 1-B (Korhonen et al. 2007) does so by superimposing synthetic magnetic anomalies computed from an age map of the ocean floor (Müller et al. 1997). This age map is derived from a self-consistent plate tectonic evolution model and, indirectly, from isochrons picked on the same marine magnetic data used in WDMAM. Because no attempt was made to adjust the synthetic anomalies to the observed ones, the result is often dominated by the synthetic anomalies whose amplitudes poorly fit that of the observations. Conversely, the Earth Magnetic Anomaly Map EMAG-2 (Maus et al. 2009) interpolates between sparse marine magnetic data using directional gridding and extrapolation based on the age map of the seafloor (Müller et al. 2008), but the result displays artificially elongated features due to the improper interpolation of anomalies unrelated to seafloor spreading (associated, for instance, to seamounts).

We adopt a strategy similar to that of the WDMAM version 1 and complement the existing marine magnetic data by building a realistic model of the magnetic anomalies caused by seafloor spreading. We made two significant improvements over WDMAM version 1: we take into account past plate motions in order to model realistic remanent magnetisation vector directions, and we adjust the synthetic magnetic anomalies to the observed ones. Our realistic modelling of the marine magnetic anomalies can be described in three steps (see also Figure 1 of Dyment et al. 2015).

In a first step, we computed magnetisation vectors in a way similar to Dyment and Arkani-Hamed (1998a), using

- the ocean floor age map (Müller et al. 2008), regionally corrected using the models by Nakanishi et al. $(1989,1992)$ and Nakanishi and Winterer (1998) for the Mesozoic lithosphere of the Northwestern Pacific Ocean and the model by Patriat (1987) and Dyment (1993) in the Madagascar and Crozet Basins, Indian Ocean;

- the relative plate motions (Royer et al. 1992) and the apparent polar wander path for Africa (Beck 1994);

- a geomagnetic polarity time scale (Cande and Kent 1995); and

- a simple hypotheses on the magnetised sources, i.e. a flat, 1-km-thick layer with top $5 \mathrm{~km}$ below sea level, bearing a $\pm 10 \mathrm{~A} / \mathrm{m}$ magnetisation.

We used these magnetisation vectors and the CM4 main field model (Sabaka et al. 2004) for year 1990 to forward model magnetic anomalies at sea level altitude and intervals of $0.01^{\circ}$ (about $1.1 \mathrm{~km}$ of latitude, less in longitude). To this end, we consider a distribution of equivalent point sources, for which the magnetisation intensity is weighted by the actual surface of the corresponding bin, and compute their magnetic anomaly following Dyment and Arkani-Hamed (1998b).

In a second step, we adjust this model to the existing marine magnetic anomaly data, in order to make it 
consistent with these data. To do so, we extract synthetic magnetic anomalies along the ship tracks, at locations where real data are available, and we compare quantitatively the measured and computed anomalies on 100-, 200- or 400-km-long sliding windows. The size of the sliding windows depends on the spreading rate: for halfspreading rates (as given by Müller et al. 2008) slower than $25 \mathrm{~km} / \mathrm{Ma}, 100-\mathrm{km}$-wide windows are sufficient to observe variations (i.e. several reversals) within each window; for half-rates faster than $50 \mathrm{~km} / \mathrm{Ma}, 400-\mathrm{km}$ wide windows are required to achieve the same goal; for intermediate rates, $200-\mathrm{km}$-wide windows are adopted. Among possible comparison criteria, we discard the maximal range as too dependent on local values and possible outliers, as well as the correlation and coherencythe geographical adjustment between model and data being too approximate-to favour the standard deviation around the mean value. The ratio between the standard deviations of observed and modelled anomalies on each sliding window represents an estimate of the magnetisation ratio at the origin of the anomalies(i.e. after multiplication by the magnetisation of $10 \mathrm{~A} / \mathrm{m}$ used in the forward model, an estimate of the equivalent magnetisation under the considered magnetised source geometry). Because the simple source geometry adopted here is far from realistic, this equivalent magnetisation can be interpreted in terms of variation of the magnetisation, the depth and the thickness of the source layer. A similar effort with a more realistic source geometry has been attempted and is presented elsewhere, providing an equivalent magnetisation interpreted in terms of magnetisation and magnetic layer thickness (Dyment et al. 2015).

In a third step, we interpolated the ratio of standard deviations (assigned to the average location of each sliding window) on a grid covering the whole oceanic domain. This ratio is the adjustment factor by which the synthetic magnetic anomalies were multiplied to be adjusted at best to the available data. The adjusted model was computed over all oceanic areas and retained for our WDMAM candidate in oceanic regions lacking data. A proper representation of the magnetic anomalies over oceanic areas consists of superimposing the available corrected marine magnetic data to this realistic model. All these types of marine data are displayed on the final grid at $0-\mathrm{km}$ altitude above the WGS84 datum.

\section{Continental data sets}

We considered the data sets and compilations whose different specificities are summarised in Table 1. Some of these data were already used for the first release of WDMAM (Korhonen et al. 2007), but there are also new data sets that were not included in the first version (e.g. Morocco) or were not available (e.g. Algeria, Romania).
Some of them are partially redundant, and we discuss below how we deal with the overlapping areas. Despite great efforts collecting data, the overall coverage remains especially sparse over Africa and South America. Unfortunately, the data sets are also missing over Western Europe where for few countries data exist but are not available (e.g. Belgium, Netherlands, Switzerland) and Central Europe (e.g. Bulgaria, Moldavia, Poland). No data were provided for New Zealand or Indonesia. Western part of the Indian grid that was constructed from ground stations has not been considered in this version. Northern India is not covered by the data. A data set has been recently made available over Afghanistan, but it is not included yet in this version of the grid. There were several versions for Russian data sets that have been tested. We used the most recent. Also, the fifth version of the Australian compilation (Milligan et al. 2010) has been preferred to previous version. Finally, we used a recently revised version of the North American compilation (Ravat et al. 2009). In the first version of the WDMAM, the compilation of Wonik et al. (1992) covered most of Europe. Unfortunately, the datum associated with this compilation is unknown, resulting in an erroneous location of the anomaly patterns. We used as little as possible this compilation in the new version of the map. Instead, we collected the national compilation and rebuilt a grid over Europe. In particular, over Western Europe, we used national grids for Portugal, Spain, France, Italy, Austria, Germany and UK. As for the first version of the WDMAM candidates (Hamoudi et al. 2007; Maus et al. 2007), three data sets of low resolution were added to fill the most important data gaps before constructing the final grid (See Getech data set - index $=46$ ). In the remaining area where no data were available, the gaps have been filled by synthetic data estimated from the lithospheric field model GRIMM_L120 (Lesur et al. 2013) derived from satellite data.

The processing technique applied on individual data set is the same as for the candidate (Hamoudi et al. 2007) of the first WDMAM version. The reader should report to this publication for more details. The data quality over each data set is difficult to estimate as complete metadata are rarely available. Most provided compilations result from putting together smaller surveys that were flown at various altitudes and epochs. In some compilations, these individual panels were not properly upward continued to a common altitude. For other compilations, this altitude and epoch information is provided but as a general rule, the mean altitudes, or the mean terrain clearances, are not known. Furthermore, the panels inside each individual data set have been reduced with IGRF/DGRF-like models or alternatively with local polynomials; but in most cases, it is difficult to find out which model was used to reduce the data. However, we had no other choice but to 
Table 1 Data sets, their statistics and sources, used in the WDMAM_2 compilation

\begin{tabular}{|c|c|c|c|c|c|c|}
\hline Name & N & Mean & Stdev. & Min/max & Index & Sources \\
\hline Marine data & $7,193,664$ & -4 & 110 & $-2057 / 2627$ & 11 & Ishihara, T., J. Luis, M. Catalan and Y. Quesnel (t-ischihara@aist.go.jp) \\
\hline \multirow[t]{2}{*}{ France } & \multirow[t]{2}{*}{$1,488,585$} & \multirow[t]{2}{*}{0.54} & \multirow[t]{2}{*}{32.00} & \multirow[t]{2}{*}{$-156 / 396$} & \multirow[t]{2}{*}{12} & Le Mouël., Ann. Geophys., 26(2), 1970 \& Courtillot et al., \\
\hline & & & & & & Earth Planet Sci. Lett., 47, 1980 \\
\hline Italy & 439,320 & 11.8 & 59.02 & $-435 / 588$ & 13 & Chiappini et al., Annali di Geophysica, 45(5), 2000 \\
\hline Spain & 494,171 & 0.02 & 16.66 & $-62 / 184$ & 14 & Socias, Earth Planet Sci. Lett., 105, 1991 \\
\hline Germany & 20,815 & -2.62 & 36.70 & $-96 / 171$ & 15 & Gerald.Gabriel@liag-hannover.de \\
\hline Austria & 5294 & 12.6 & 26.12 & $-35 / 137$ & 16 & GSA, (http://www.geologie.ac.at/) \\
\hline Fennoscandia & $3,505,857$ & -30 & 154 & $-580 / 1500$ & 17 & J. Korhonen, GTK (http://en.gtk.fi/) \\
\hline United Kingdom & 119,509 & -2.71 & 121 & $-962 / 1342$ & 18 & British Geological Survey, NERC, IPR/132-01CT \\
\hline Portugal & 151,142 & -10 & 33 & $-111 / 219$ & 19 & Miranda et al., Earth Planet Sci. Lett., 9, 1989 \\
\hline \multirow[t]{2}{*}{ Romania } & \multirow[t]{2}{*}{100,092} & \multirow[t]{2}{*}{19} & \multirow[t]{2}{*}{70} & \multirow[t]{2}{*}{$-144 / 418$} & \multirow[t]{2}{*}{20} & Dr. L. Besutiu, Institute of Geodynamics, Romania \\
\hline & & & & & & (http://www.geodin.ro/) \\
\hline Japan (2007) & 91,094 & -42 & 73 & $-401 / 416$ & 21 & http://www.ccop.or.th/ \\
\hline East Asia & 958,040 & -28 & 68 & $-509 / 763$ & 22 & http://www.ccop.or.th/ \\
\hline Russia (vsegei) & $1,688,415$ & 0.9 & 158 & $-2274 / 5658$ & 23 & T. Litvinova, VSEGEI (http://www.vsegei.ru/en) \\
\hline Djibouti & 266,171 & -2.8 & 85 & $-293 / 241$ & 24 & Courtillot et al., Earth Planet Sci. Lett., 47, 1980 \\
\hline Middle-East (2007) & $1,812,464$ & 0.56 & 73 & $-904 / 690$ & 25 & http://members.casema.nl/errenwijlens/itc/aaime/ \\
\hline India & 92,784 & 18.5 & 82 & $-341 / 427$ & 26 & GSI, http://www.portal.gsi.gov.in/ \\
\hline Circum-Arctic & $3,258,710$ & -4 & 99 & $-699 / 2754$ & 27 & $\begin{array}{l}\text { Gaina, C., Werner, S.C. and the CAMP- GM group, NGU Report } \\
2009.010\end{array}$ \\
\hline Guadeloupe & 353,672 & 24 & 46 & $-76 / 190$ & 28 & IPG Paris, France, (http://www.ipgp.fr/) \\
\hline French Guiana & 99,066 & -3.9 & 72 & $-283 / 143$ & 29 & BRGM, France (http://infoterre.brgm.fr/) \\
\hline Bahama-Cuba & 329,975 & 0.9 & 75 & $-410 / 681$ & 30 & Batista-Rodriguez, et al., Geophysics, 72(3), (doi:10.1190/1.2712425) \\
\hline Nure-Namag & $2,012,238$ & -11 & 123 & $-3667 / 3998$ & 31 & Ravat, et al., USGS Open-file Report 2009-1258 \\
\hline Algeria & $2,126,820$ & 0.2 & 48 & $-189 / 274$ & 32 & Ministry of Energy and Mines, Algiers, Algeria \\
\hline Morocco & 394,179 & -1.4 & 43 & $-194 / 207$ & 33 & Ministry of Energy and Mines, Rabat, Morocco \\
\hline Nigeria & 132,407 & 26.3 & 40 & $-163 / 177$ & 34 & Nigerian Geological Survey, Abuja, Nigeria \\
\hline Argentina-Shelf & $1,497,947$ & -9 & 45 & $-150 / 318$ & 35 & Max et al., Marine Petroleum Geology, 16, 1999. \\
\hline Argentina-Inland & 34,134 & -1.5 & 104 & $-3152 / 1173$ & 36 & SEGEMAR, http://www.segemar-cba.com.ar/ \\
\hline Australia & $7,089,164$ & -41.2 & 137 & $-1674 / 3360$ & 37 & (Milligan et al. 2010), Geosciences Australia, \\
\hline & & & & & & http://www.ga.gov.au/data-pubs/maps \\
\hline South African DC & 427,270 & -3.2 & 120 & $-1788 / 2030$ & 38 & SADC, http://www.sadc.int/ \\
\hline Uganda & 123,020 & -83 & 116 & $673 / 301$ & 39 & Ministry of Energy and Mineral Development, Uganda \\
\hline Mozambique & 419,248 & 159 & 171 & $-409 / 1007$ & 40 & Geologian Tutkimuskeskus, Espoo, Finland \\
\hline SaNaBoZi & 83,380 & 77 & 109 & $-203 / 756$ & 41 & SADC, http://www.sadc.int/ \\
\hline Acad Vernadsky & 69,123 & 170 & 166 & $-180 / 426$ & 42 & Orlyuk et al., St Petersburg, July 8th-11th, Russia, 2008 \\
\hline Admap & $56,125,472$ & 15 & 104 & $-776 / 1371$ & 43 & https://www.bas.ac.uk/data/our-data/maps/map-catalogue \\
\hline Marine Model & 901,539 & -5 & 152 & $-1662 / 1639$ & 44 & Dyment et al., EPSL, 430, 2015 \\
\hline Eurasia (2007) & 540,898 & -2 & 102 & $-891 / 819$ & 45 & GSC, http://gsc.nrcan.gc.ca/ \\
\hline Getech (2007) & $1,038,080$ & 1 & 59 & $-1024 / 536$ & 46 & GETECH, http://www.getech.com/gravity-magnetic/ \\
\hline GRIMM_L120 & $3,645,256$ & 8 & 55 & $-235 / 580$ & 47 & Lesur et al., Solid Earth, 4, 2013 \\
\hline
\end{tabular}

estimate these altitudes and reference model and continue the data processing with the estimated values. Finally, the compilations are provided in different format, coordinate systems and projections, and we did our best to account for these. Overall, the final patch-worked compilations are prone to mismatch in anomaly shapes and strengths that 
may easily be confused with magnetic anomalies. Moreover, the lack of absolute reference makes it difficult to restore the large wavelengths: these should be regarded with caution. A complete processing was applied to each data set, except for Eurasia, India and Mexico that were provided partially processed.

The data sampling intervals are not homogeneous but are easy to recover. They vary from about 50 and $30 \mathrm{~km}$ for India and Getech grids, respectively, to 1-km spacing for North America, Australia, Europe and Russia. Other available data sets are of sufficient resolution-e.g. Algeria and Morocco, with $5-\mathrm{km}$ resolution. One must notice that the resolution does not necessarily correspond to the grid spacing. It may not even be homogeneous over provided compilations. As a result, we may see some regions free of short wavelength magnetic anomalies simply because of the lack of resolution.

All data over continental areas are upwardly continued to 5 - $\mathrm{km}$ altitude relative to the WGS84 datum.

\section{Merging}

The data sets included in the WDMAM often overlap, sometimes over very large areas. All data are not of the same quality; therefore, it is advisable to define an order of preference between data sets. In particular, the data set used to fill the gaps (see Table 1 , indices $=45,46,47$ ) are either of poor quality or low resolution. National grids derived from aeromagnetic data are usually all of similar quality. Marine data are generally of lower quality than airborne data despite their re-levelling (Quesnel et al. 2009). We therefore limit the overlap between two adjacent compilations to few tens of kilometres and, in some cases, avoid any overlap.

The precedence we defined follows a numbering that is given in Table 1 . The choice of preference order should, in principle, reflect the data quality, but this is not necessarily the case. For example, the data set with the highest preference order is the marine data set (index $=11$ ), not because of its quality but simply because marine data are given at sea level, whereas other data compilations are upwardly continued to $5-\mathrm{km}$ altitude. Similarly, the Australian data set, which is known for its homogeneity and quality, has a low preference order (index $=37$ ), but the data set remains unmodified because it is isolated from other data compilations.

The permitted overlaps between data sets is typically 5 cells of the final grid (i.e. $25 \mathrm{~km}$ ); if the overlap is larger than 5 cells, the data with lower priority order are cutout. Exceptions to this 5-cell rules are the marine data, the marine model data and the long wavelength synthetic data derived from the GRIMM_L120 magnetic model. For this type of data, no overlap is permitted.

Before merging the individual data sets-that now have limited overlap but are not necessarily on a regular grid, they are interpolated using again the GMT function nearneighbor with either a $5-\mathrm{km}$ search radius or $7-\mathrm{km}$ search radius - depending on data spacing, and requiring data in at least two quadrants. An exception to this are the data sets from India and Middle East that require a search radius of $10 \mathrm{~km}$. After these steps, all data sets are on a regular, $5-\mathrm{km}$ cell size, grid.

The merging process, in an area of overlap, defines for each grid point a weighted average value. The weighting and averaging scheme follows three rules:

1. Each point of the grids is given a starting weight. This weight depends on the position of the point in its grid. For a data grid and a specific point in that grid, a $11 \times 11$ mask is centred on that point and the number $n$ of mask nodes belonging to the data grid is calculated. The starting weight for the point is then

$$
w=\left(\frac{n}{121}\right)^{2}
$$

Typically, a point in the centre of a grid has a weight $w=1$, but it is often less than $w=0.25$ for a point on the edge of a grid.

2. Let us assume there is two overlapping grids: grid $\alpha$ and grid $\beta$. These grids are merged in a single grid where the points in the overlapping area take the value

$$
\text { value }=\left(\frac{w_{\alpha} \cdot \text { value }_{\alpha}+w_{\beta} \cdot \text { value }_{\beta}}{w_{\alpha}+w_{\beta}}\right),
$$

where value ${ }_{\alpha}$ and value $\beta$ are the grid point values in grids $\alpha$ and $\beta$, respectively. $w_{\alpha}$ and $w_{\beta}$ are their associated weights. The weight for the obtained value is then $w=w_{\alpha}+w_{\beta}$ that must be calculated to merge further data to the grid.

3. All grids are successively merged to cover the full Earth. The indices of points in overlapping area are set to index $=0$.

This merging process suffices to smooth sharp discontinuity between adjacent grids, while limiting to the edge of each grid possible degradation of the data quality. At the end of the merging process, we obtain a compilation of all available data on a global $0.05 \times 0.05$ degree cell size grid-(i.e. roughly $5 \times 5 \mathrm{~km}$ cell size), with no missing values.

\section{Long wavelength handling}

When compiling a magnetic data set of national extent, scientists try to handle properly the longest magnetic 
anomaly wavelengths. However, it is practically impossible to account for the anomalies of neighbouring countries, and therefore the long wavelengths of a merged grid of several aeromagnetic and marine data sets are necessarily wrong. In the WDMAM compilation, we use a geomagnetic lithospheric model derived from satellite data to correct for the wavelengths corresponding to $\mathrm{SH}$ degrees 16 to 100 . Larger wavelengths, for $\mathrm{SH}$ degrees 1 to 15 , correspond to the core field. They are filtered out. For wavelengths shorter than $\mathrm{SH}$ degree 100, no accurate information is available from the satellite data and we have to rely exclusively on aeromagnetic and marine data even if we have little trust on their long wavelength content.

In principle, extracting the longest wavelengths from a global compilation to replace them by a satellite derived magnetic field model is straightforward. In its application, however, this process is not so easy to handle. To extract the long wavelengths, one has to build a $\mathrm{SH}$ model of the magnetic lithospheric field; the long wavelengths correspond to the low-degree Gauss coefficients. We describe in the next section the way such a spherical harmonic model is built. This requires slightly regularising the inversion, which implies that a smoothing parameter has to be set arbitrarily. It follows that we can never be fully confident in the validity of the derived model. To circumvent this difficulty, we use a different approach that does not require the arbitrary setting of a smoothing parameter.

In a first step, we calculated synthetic values of the largescale lithospheric anomaly field using the GRIMM_L120 lithospheric field model (Lesur et al. 2013) up to $\mathrm{SH}$ degree 120. This was done on the same grid as the WDMAM and using the same reference model: CM4 (Sabaka et al. 2004) for year 1990. Then, the synthetic grid and the grid obtained as an output of the WDMAM merging process were sub-sampled on $0.5 \times 0.5$ degree cell size grids using the GMT function blockmedian. The sampling point positions were then calculated in the geocentric system of the coordinate. In a second step, we calculated for both $0.5 \times 0.5$ degree cell size grids a $\mathrm{SH}$ expansion of the total intensity anomaly. We used the maximum $\mathrm{SH}$ degree 300 for the grid output of the merging process and only $\mathrm{SH}$ degree 130 for the grid corresponding to the GRIMM_L120 model. These two SH expansions are then truncated to SH degree 100 and their differences calculated. Finally, these differences are subtracted from the merged grid, giving the final candidate grid for the WDMAM.

It shall be noted that the SH expansion of the anomaly field of a lithospheric model is not the $\mathrm{SH}$ expansion of this model. In particular, these two SH expansions do not have the same $\mathrm{SH}$ degree bounds. As a result, the long wavelengths of the WDMAM data are different from the long wavelengths of the magnetic field model GRIMM_L120. They are also different from the long wavelengths of the anomaly field calculated from the GRIMM_L120 model truncated to SH degree 100.

\section{The WDMAM grid}

We built the WDMAM candidate grid where each sampling point is given as longitude and latitude in geodetic system of coordinates (i.e. WGS84 datum). For each sampling point is also given the value output of the merging process with the difference between the SH degree 100 expansions subtracted, the index corresponding to the type (or origin) of data entering the compilation and the values of the SH degree 100 expansion of both the merged grid and the GRIMM_L120 anomaly data. These latter 2 values are provided such that the user can re-estimate the WDMAM grid values before the long wavelength filtering and use any other field model to correct the long wavelengths.

The resulting grid of magnetic anomaly field, relative to the CM4 field model for 1990, is presented in Fig. 1. The grid of indices is shown in Fig. 2.

\section{High-resolution lithospheric magnetic field model}

The WDMAM is a grid of 25,927,200 total intensity anomaly values, that in principle should allow to build a magnetic field model to a maximum $\mathrm{SH}$ degree larger than 5000. However, there is very little interest in building such a model given the limited accuracy of the grid. We nonetheless built a model to $\mathrm{SH}$ degree 800 in order to study the spectral content of the grid and also its local variations.

To build this model, we first reduced the size of the data set to a $0.2 \times 0.2$ degree cell size grid using the GMT function blockmedian applied on the compilation. We are therefore left with a grid of 1,620,000 total intensity anomaly values. Each data point position was evaluated in geocentric spherical coordinates system accounting for the difference in altitude between marine and continental data. Total intensity anomaly values are non-linear functions of the parameters of the lithospheric model (i.e. the Gauss' coefficients), and an iterative process was set to define the model. The lithospheric field model was fitted to this data set through a least squares algorithm using an L2 measure of the misfit. The data were given weights proportional to the sine of their colatitudes. The posterior distribution of the residuals did not show any evidence that an alternative measure, as an Huber norm, should be used. At degree 800, a magnetic field model of the lithosphere has 641,600 parameters, and therefore a gradient algorithm has been used to derive the model. Since there is a significant amount of noise in the data, the inversion process needed to be regularised to avoid propagation of this noise in the model along the direction perpendicular to the main field vector. This type of difficulty 


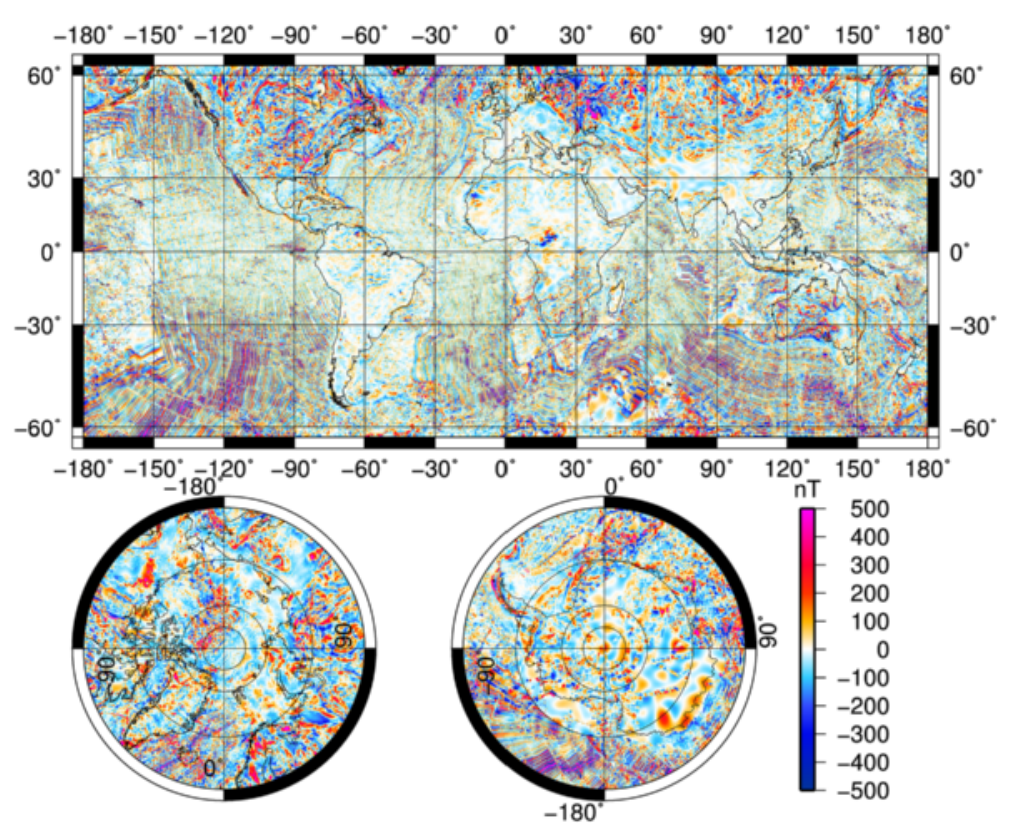

Fig. 1 Global map of the Earth's magnetic anomalies relative to the CM4 main field model for year 1990

is known in the geomagnetic community as the "Backus effect". The regularisation was achieved by minimising the integral over the sphere of the strength of the field model component perpendicular to main field directioni.e. for the WDMAM the CM4-1990. It is controlled through a regularisation parameter $d$. The model is defined on the reference spherical surface of radius $6371.2 \mathrm{~km}$.

The power spectrum of our model is shown in Fig. 3 for few values of the regularisation parameter, together

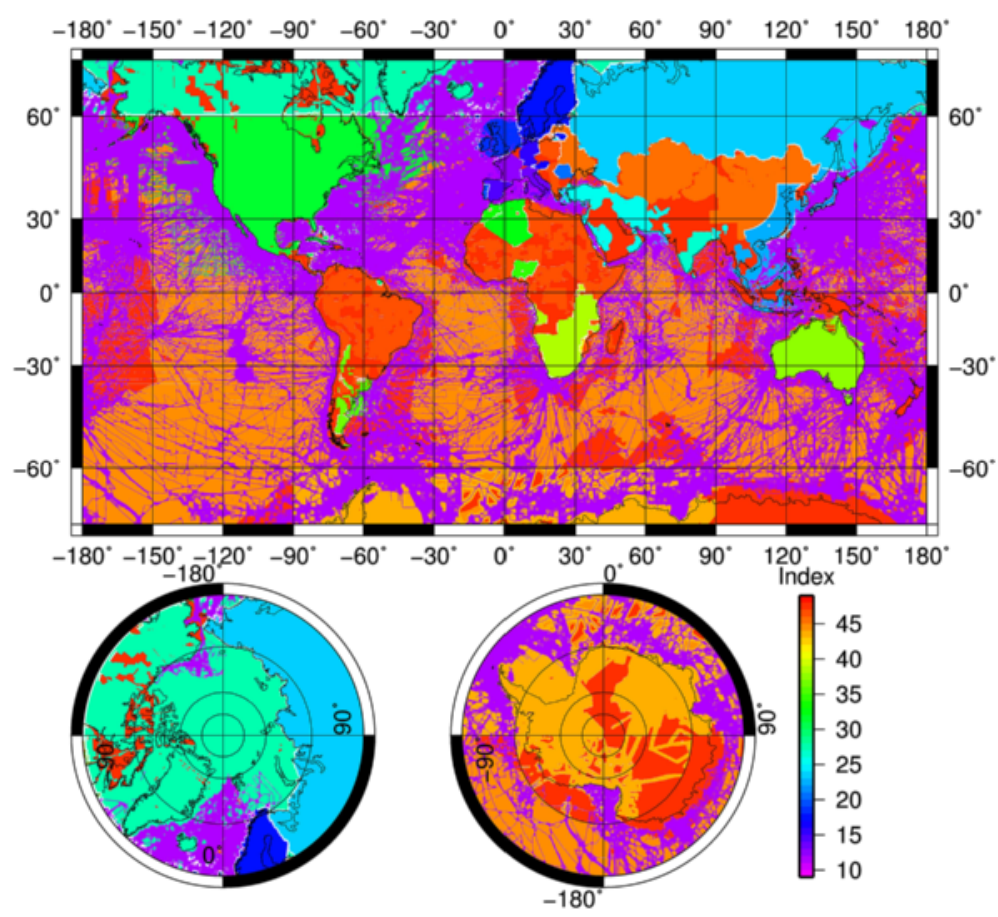

Fig. 2 Global map of the data set indices ranging from 11 to 47. The list of contributing data sets with their associated indices is given in Table 1 


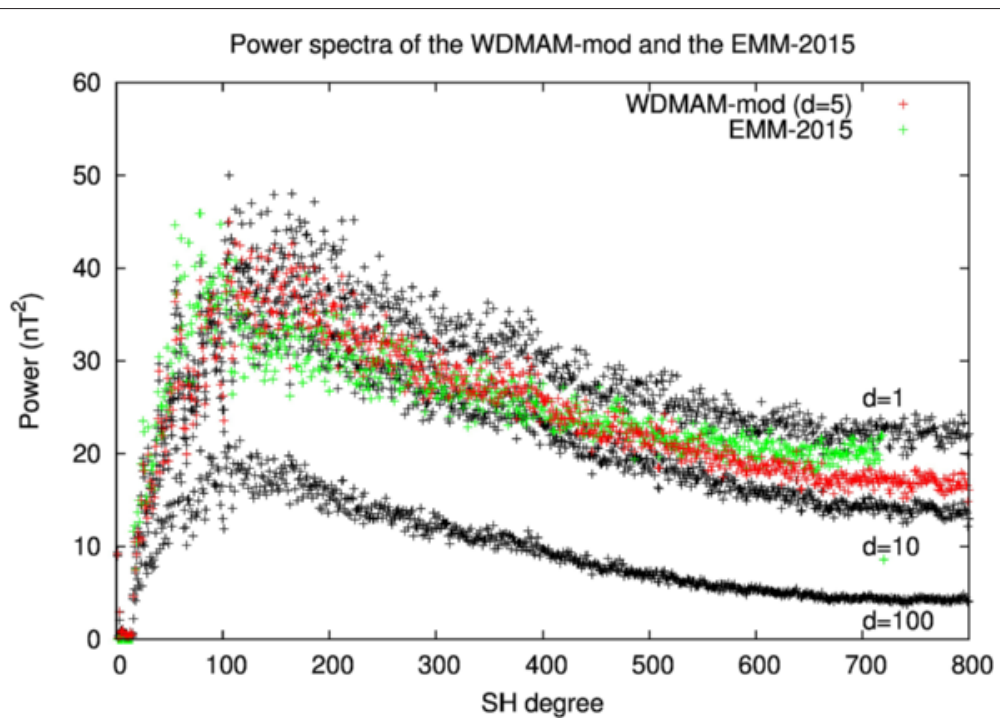

Fig. 3 Global power spectra of models of the Earth's lithospheric magnetic field calculated at a reference radius of $6371.2 \mathrm{~km}$. The spectra for models with different regularisation parameter values $(d=1,10,100)$ are presented. The spectrum for the chosen parameter $d=5$ is shown in red and the spectrum of the EMM2015 is shown in green

with the EMM2015 model (https://www.ngdc.noaa.gov/ geomag/EMM). The final value chosen for the regularisation parameter is $d=5$. This is set exclusively by visual inspection of the final map, trying to limit as much as possible spurious elongation of the anomalies in the north/south direction near the magnetic dip equator. A more precise adjustment of this regularisation parameter is possible in the interval [ $4: 5]$, but the characteristics of the model would not be much different. Such a precise adjustment would be however extremely time consuming and probably not worthwhile. The map associated with the model is shown in Fig. 4.

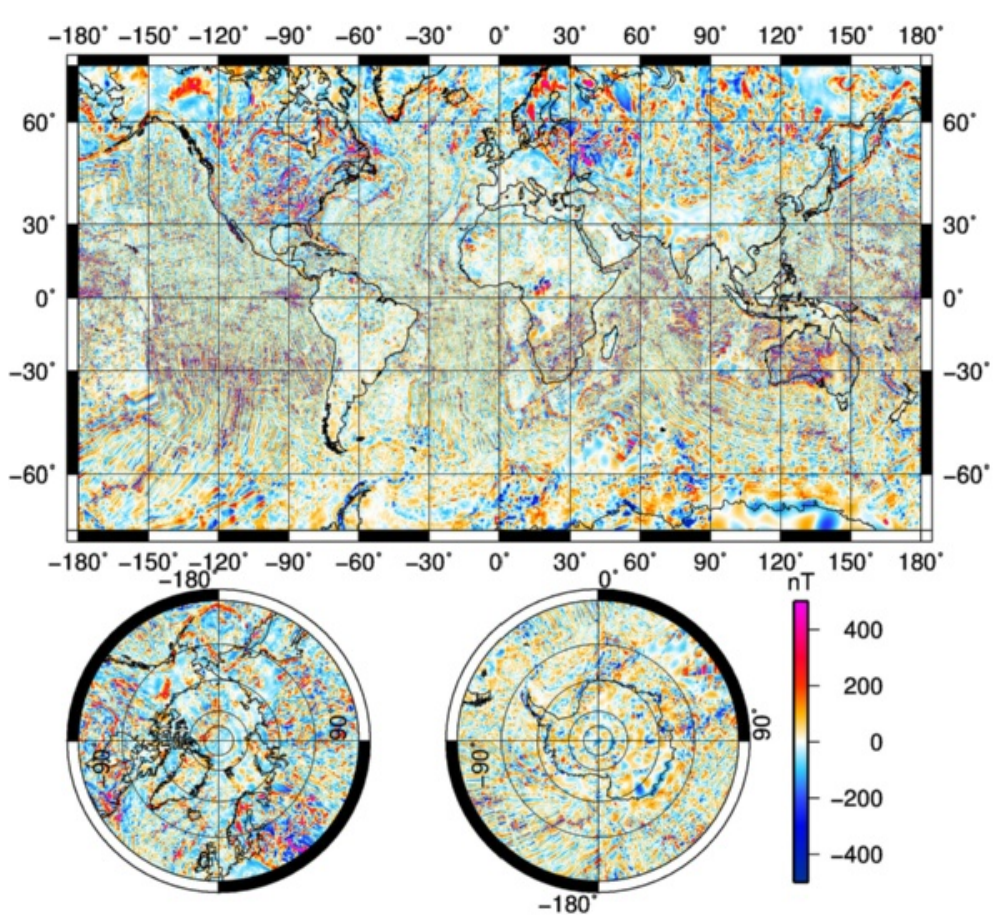

Fig. 4 Global map of the vertical down component of the model of Earth's magnetic field generated in the lithosphere and derived from the candidate to the second version of the WDMAM 
The derivation of this model allows the calculation of local spectra following the method presented by Vervelidou and Thébault (2015). The global WDMAM model is first used to compute synthetic vector measurements to SH 900 on a dense equal area grid located at the Earth's mean radius. A modelling method relying on 2D spherical local functions (Thébault 2008) is then applied to fit the synthetic measurements to an equivalent $\mathrm{SH}$ degree 900 within 2000 caps homogeneously distributed on the reference sphere. The power spectrum is computed for each cap, and the cutoff harmonic degree is defined as the first degree from which the power spectrum value is lower than the misfit value between the regional model and the synthetic measurements. The geographical distribution of the cutoff SH degree shown in Fig. 5 provides a regional estimation of the model information content.

\section{Results and discussion}

The predominant feature of the WDMAM candidate map shown in Fig. 1 is the strength of the lithospheric anomaly field over Siberia and North America. These large values of the anomaly field strength contrast with much weaker values in Africa, South-America, Western Europe and some parts of Asia.

In Western Europe, the weakness of the field is a real feature of the anomaly field. Here, the data sets are of the highest quality over most of the countries. An exception is the lack of data over the Balkans where the anomaly field should be relatively large as suggested by the eastern part of the Italian compilation. Similarly, there is no data over Switzerland and Netherlands, but only weak fields are expected over the latter country. Other large anomaly values are visible over Scotland but, overall, the anomaly field remains weak. This area of weak field is limited to the east by the Tornquist-Teisseyre line separating the Precambrian East European Platform and the Palaeozoic West European Platform.

In other parts of the world, the weakness of the anomaly field is due to two factors. The first dominant factor is the lack of aeromagnetic data. One can immediately see that in Australia and Southern Africa, two areas covered with high quality airborne surveys, the anomaly field is relatively small scale and strong. We should expect the same over South America but there, outside few small surveys on the western limit of Argentina, there is practically only over-smoothed and decimated data values. There is simply no available data over the Arabic Peninsula, Himalayan area and Oceania. The second factor is the weakness of the main magnetic field in equatorial areas and large parts of the Southern Hemisphere. The main field weakness precludes the generation of strong induced magnetisation in the rocks and therefore the generation of large anomaly fields. To evaluate the effect of the weakness of the inducing field, we divided the WDMAM anomaly values by a dimensionless factor: $\mathcal{J}_{D}$, equal to the main field strength at the data point over $45,000 \mathrm{nT}$. The $\mathcal{J}_{D}$ factor ranges

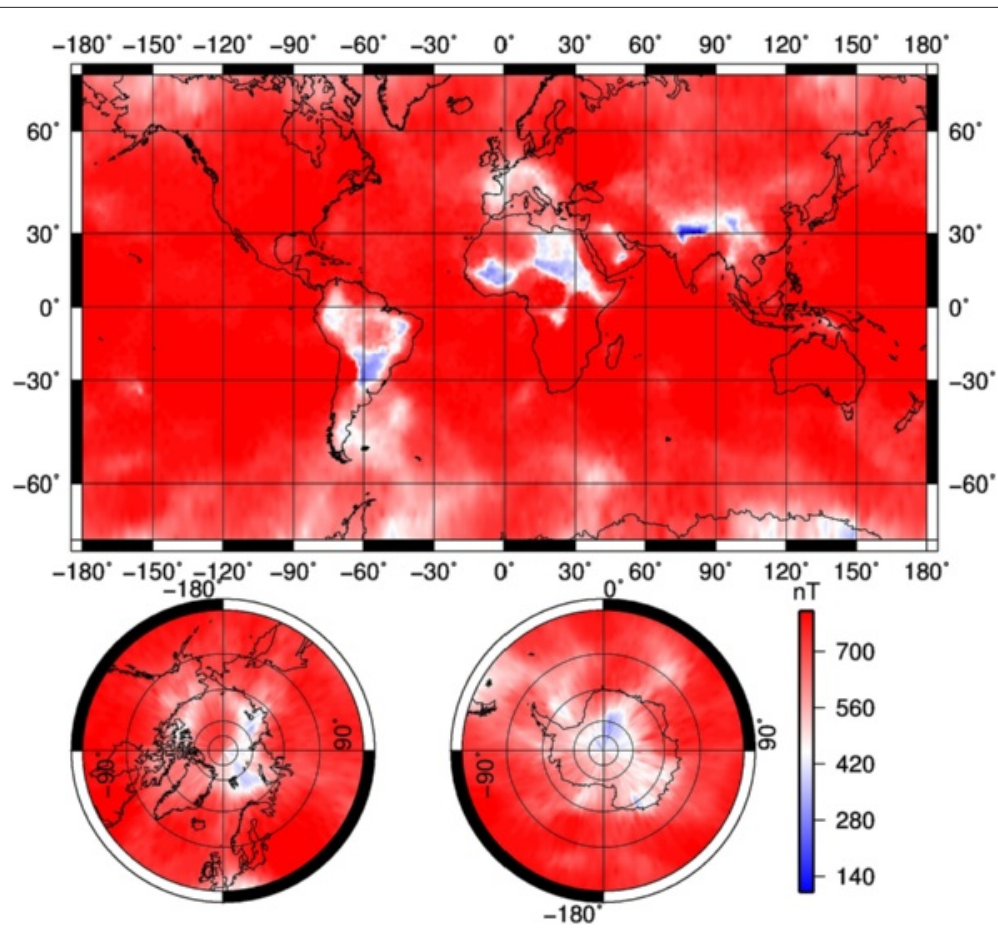

Fig. 5 Map of the true minimum wavelength of the magnetic field model as a function of the location, presented as a maximum SH degree 
between 0.5 and 1.5 and is mapped in Fig. 6. The anomaly field divided by the $\mathcal{J}_{D}$ factor is shown in Fig. 7. This operation is meaningful if it is assumed that the anomaly field is essentially due to an induced magnetisation. This hypothesis is clearly wrong over oceanic areas, leading to a strong signal in the Southern Pacific and Southern Atlantic areas. The signal is weakened over Siberia and Canada, with values of the scaled anomaly field of the same order than Australia and smaller than for the South African region. In South America, the signal is significantly increased and will probably reach values as large as the northern part of the continent if data of acceptable quality are provided. The scaled anomaly field seems to be of the same amplitude over the different cratons, but it remains surprisingly weak over Northern Africa, and very strong over Scandinavia and Eastern Russia. A strong remanent magnetisation is possibly one of the reason for these latter features.

Three significant improvements between WDMAM versions 1 and 2 are worth noting in oceanic areas. First, new data have been added to the data base built by $\mathrm{T}$. Ishihara and M. Catalan (personal communication). Second, the synthetic magnetic anomaly model now takes into account the plate tectonic evolution to simulate the remanent magnetisation vector direction, whereas this vector was parallel to the present-day field in the previous version. Third and most noticeable is the adjustment of the synthetic magnetic anomaly model to the data, performed by comparing the standard deviation of synthetic and observed anomalies on sliding windows along the real ship tracks. This adjustment leads by construction to much smoother transitions between the oceanic areas where sufficient data are available to define a grid and those where the model has to be used instead. This makes the WDMAM version 2 map more realistic in such areas. It should be noted that, because various processing applied by different data compilations result in considerable and variable smoothing of the seafloor spreading magnetic anomalies, we gave precedence to the original data and the synthetic anomalies adjusted on these original data over these compilations. Observed and synthetic anomalies in oceanic areas can easily be separated using the index given as the fourth column of the WDMAM version 2 table. Various errors arise from imperfections remaining in the seafloor age map. We corrected the age map in the Northwestern Pacific Ocean and in the Madagascar and Crozet Basins (Indian Ocean) where the seafloor age map of Müller et al. (2008) defines isochrons at high angle $\left(90^{\circ}\right.$ in the Madagascar Basin!) with respect to the unambiguously determined ones in these areas, pointing to errors in the age map. Other places where obvious errors, generally caused by careless interpolation, have been depicted in the age map by our modelling, have been masked in the final synthetic anomaly model. Similarly, places where these synthetic anomalies are likely not representative are not considered. This includes the oceanic plateaus, aseismic ridges, and the Cretaceous and so-called Jurassic Quiet Zones where the model generates no magnetic anomaly. In the areas where both marine data and synthetic data are unavailable, the gaps are filled

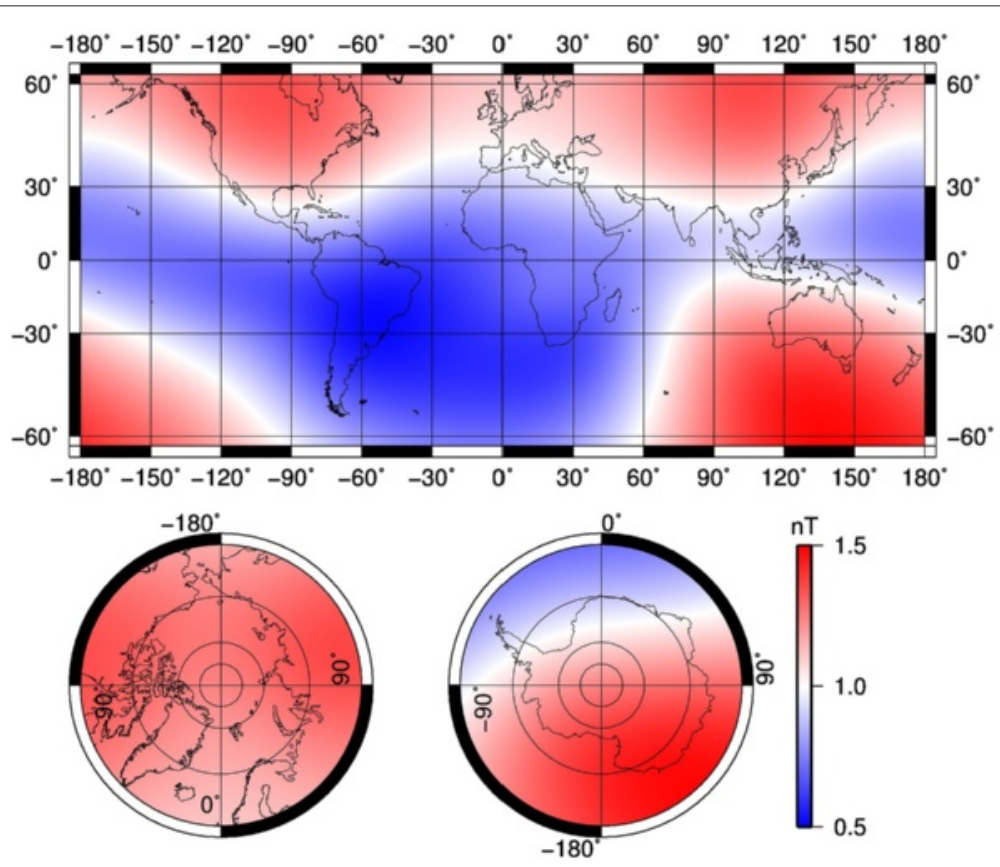

Fig. 6 Map of the $\mathcal{J}_{D}$ factor. The $\mathcal{J}_{D}$ factor is dimensionless and consists of the ratio of the CM4 core field strength for 1990 over $45,000 \mathrm{nT}$ 


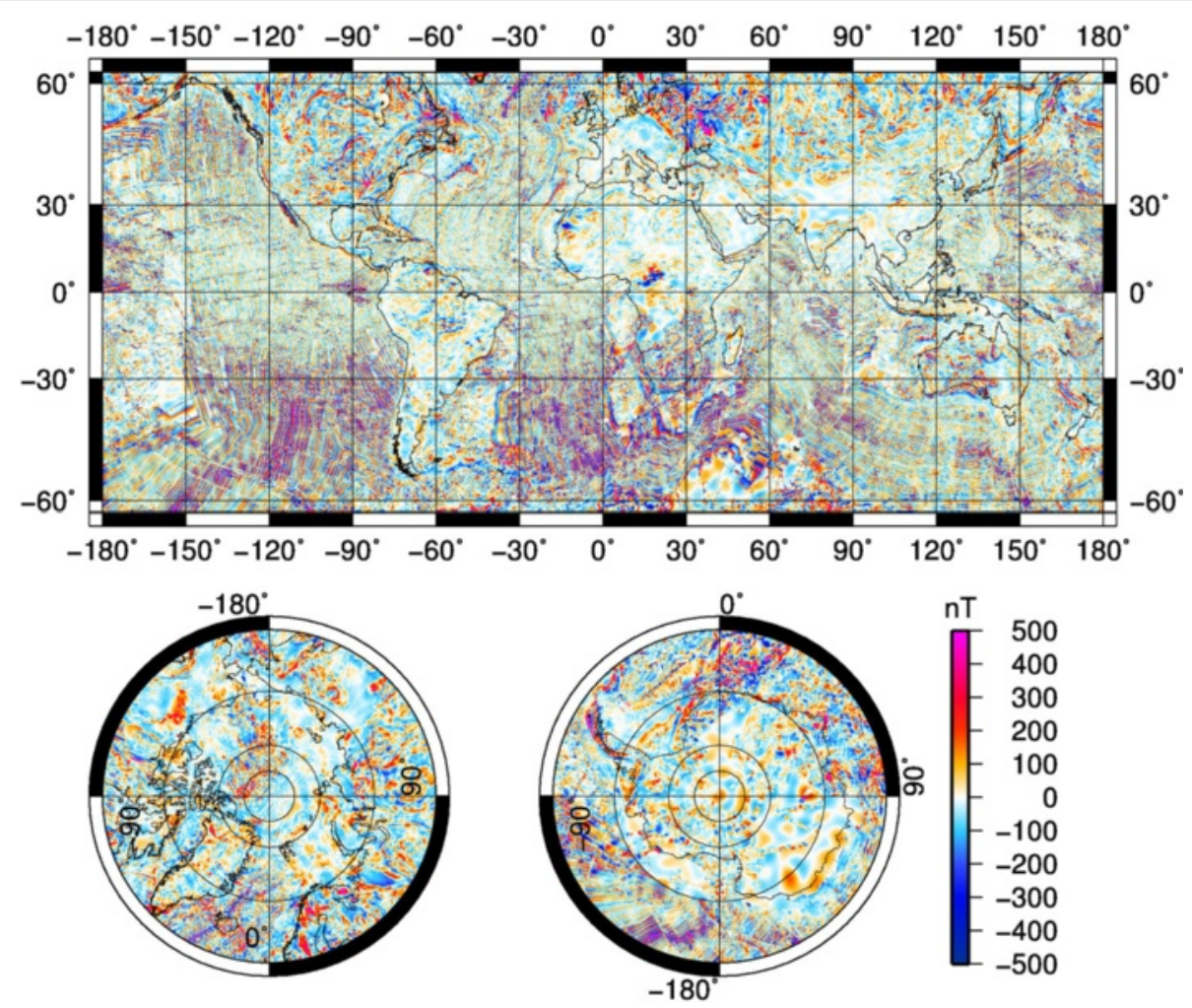

Fig. 7 Global map of the Earth's magnetic anomalies relative to the CM4 main field model for year 1990, divided by the $\mathcal{J}_{D}$ factor

with anomaly values obtained from a magnetic field models derived from satellite data. A final advantage of our approach is the derivation of a global grid of adjustment factors derived from the comparison of observed and synthetic anomalies. Once corrected for the constant magnetisation used to compute the synthetic anomalies, this adjustment factor grid has the dimension of an equivalent magnetisation. The derivation of such a global distribution of equivalent magnetisation, computed with a more realistic source geometry considering the bathymetry and sediment thickness, has been derived and is described elsewhere (Dyment et al. 2015).

As explained before, one of the difficulties in generating the anomaly map is the integration of the robust information about the long wavelength lithospheric field derived from satellite data. We used the GRIMM_L120 model as the reference model; however, there is no doubt that significantly better models will be derived using Swarm data. We have therefore included enough information in the WDMAM grid such that the long wavelengths of the lithospheric field can be corrected again if necessary. Another point is that the long wavelength of the lithospheric field model derived from the WDMAM grid does not match the long wavelengths of the GRIMM_L120 model. This discrepancy between the original and final Gauss coefficients may be due partly to the regularisation applied when deriving the WDMAM model but also to the process we applied for replacing these long wavelengths. The latter effect is particularly large for SH degree between 95 and 100. Again, scientists not satisfied with this approach have all the necessary information to correct these long wavelengths.

The global magnetic field model was derived from the WDMAM grid through a simple least squares process that also minimise the "Backus effect". Since our model is truncated at SH degree 800, it is not singular even if the true sources of magnetic field anomalies are above the Earth reference radius of $6371.2 \mathrm{~km}$. This is why we did not find necessary to account for the flattening of the Earth to derive this model. Our model has slightly less energy than the EMM2015 at SH degree below 100 but more energy from SH degree 100 to 200. It has a spectrum with a slightly steeper slope at high SH degrees but otherwise a similar power level. A close inspection of the vertical component maps of both models shows that they differ mainly by their signals over oceans. A more precise analysis is difficult because there is no scientific publication associated with the EMM model-(only the first version of the NGDC720 model was released with a publication, see Maus 2010). The study of the local spectra of our magnetic field model shows that the model has a low cutoff $\mathrm{SH}$ degree over Antarctica, South America, Tibet and Africa 
(Fig. 5). These are regions poorly covered by aeromagnetic and marine measurements. In contrast, the maximum of information content is found for regions well covered by near-surface data or in oceans where magnetic signatures are mostly small scale. In regions such as Western Europe, the Barents Sea or Northern Greenland, the model has comparatively low spatial resolution despite the availability of dense, homogenous and good quality magnetic data sets. Thus, the resolution analysis that reflects both the quality of the input data in a region and true magnetic properties should always be analysed in combination with Fig. 2.

\section{Conclusion}

In this paper, we have described the derivation of the second version of the World Digital Magnetic Anomaly Map (WDMAM). The approach we followed differs in different aspects from the first version of the map. First, we tried to modify as little as possible the data sets and compilations provided for building this map. In particular, we always limit to a few kilometres the overlap between adjacent data sets. Second, we give the highest priority to the marine data, and therefore they are preferred to national grids that extend over oceans. This has two consequences: (1) the WDMAM over the marine area has a reference altitude of $0 \mathrm{~km}$ above the WGS84-this altitude was $5 \mathrm{~km}$ in the first version of the map, (2) All data over marine area have been interpolated in a consistent way. Third, we have filled the gap over marine area with a fully revised model of the signal generated by the oceanic floor magnetisation. Overall, the map presents a much more consistent view of the Earth lithosphere magnetic anomaly field.

Compared to the first version of the map, we have also provided further information that can be useful for the scientific exploitation of the compilation. As before, the basic information consist of a point position in latitude and longitude, the value of the anomaly field and an index that allows to find what was the original data set that enter the compilation. Marine data are at $0 \mathrm{~km}$ above the WGS84, whereas continental data are at $5 \mathrm{~km}$ above the WGS84. We also provided the value of the anomaly field associated with GRIMM_L120-that is a model derived from satellite data and representing the known large-scale lithospheric magnetic field, and also the anomaly field of the large-scale contribution of the compiled data sets. The former and latter anomaly values are the quantities that have been added and subtracted, respectively, to the collected magnetic anomaly data so that the long wavelength content of the map is as close as possible from the true lithospheric anomaly field. With these provided anomaly values, the long wavelength content of the map can be easily corrected again by the user. Another useful product is a spherical harmonic model of the anomaly map up to degree 800 . The maximum degree is not so important since, as it has been shown, in numerous areas, there is insufficient data to build an accurate model up to this wavelength. This model can nonetheless be used, e.g. to control the long wavelength content of a new data set acquired on a limited area anywhere on Earth.

The analysis of the WDMAM and the associated field model confirm the weakness and smoothness of the lithospheric magnetic field over Europe and possibly over Northern Africa. Other continental area may present a weak field, but this is likely due to the weakness of the inducing main field in these area.

Finally, we insist on the fact that building this compilation is only a small contribution compared to the enormous amount of work that was involved in collecting and processing aeromagnetic and marine data. The quality of the final compilation depends mainly on the availability of these data. Since the official release of the WDMAM during the IUGG-2015, several new compilations have been offered to the WDMAM task force. These will be incorporated soon in a new version of the WDMAM compilation: namely WDMAM v2.1. This and following versions 2.x will be computed and distributed as new data become available. The same methods described in this paper will be used, until the need for methodological improvements is required. A call for the WDMAM v3 is then likely to be issued, when decided by IAGA Div. V-mod. We therefore encourage interested colleagues or institutions to provide any other new data sets. We will be happy to incorporate (or help in incorporating) these in the compilation. Of course, the institutions or scientists providing the data sets become, if they wish, co-authors of the WDMAM.

\section{Competing interests}

The authors declare that they have no competing interests.

\section{Authors' contributions}

All co-authors have, along the last 5 years, contributed to the data collection, organisation and work leading to the final compilation. All have contributed to the redaction of this paper. MH did a significant part of the data set processing. JD and YC work focussed on the marine data sets and models. VL did the merging process, handled the long wavelength problem and, together with ET, produced the model of the lithosphere magnetic field. All authors read and approved the final manuscript.

\section{Acknowledgements}

WDMAM data contributors (see Table 1) and evaluators, together with members of the WDMAM task force, are fully acknowledged for their work and constant support. We thank also the reviewers of this paper. VL started his contribution to this work while he was at GFZ; further, a significant part of the data processing has been done while MH was a visiting scientist in GFZ and in IPGP. This work was partly funded by the Centre National des Etudes Spatiales (CNES) within the context of the project of the "Travaux préparatoires et exploitation de la mission Swarm". This is IPGP contribution 3717.

\section{Author details}

${ }^{1}$ Institut de Physique du Globe de Paris, CNRS UMR 7154, 1 rue Jussieu, 75005 Paris, France. ${ }^{2}$ Université des sciences et de la technologie, 24105 Bab Ezzouar, El-Alia Alger, Algeria. ${ }^{3}$ Helmholtz Centre Potsdam, GFZ German Research Centre for Geosciences, Telegrafenberg, 14473 Potsdam, Germany. ${ }^{4}$ LPGN, Université de Nantes, 2 rue de la Houssinière, 44322 Nantes Cedex 3, France. 
Received: 21 December 2015 Accepted: 6 February 2016

Published online: 19 February 2016

\section{References}

Beck F (1994) Courbes de dérive des pôles du Permien à l'actuel pour les continents péri-Atlantiques et Indiens: Confrontation avec les reconstructions paléogéographiques, Ph.D. thesis. Univ. Louis Pasteur,Strasbourg, France. p 222

Cande SC, Kent DV (1995) Revised calibration of the geomagnetic polarity timescale for the Late Cretaceous and Cenozoic. J Geophys Res 100 B4:6093-6095

Dyment J (1993) Evolution of the Indian Ocean Triple Junction between 65 and $49 \mathrm{Ma}$ (anomalies 28 to 211). J Geophys Res 98. doi:10.1029/93JB00438

Dyment J, Arkani-Hamed J (1998a) Contribution of lithospheric remanent magnetization to satellite magnetic anomalies over the world's oceans. J Geophys Res 103:15423-15442

Dyment J, Arkani-Hamed J (1998b) Equivalent source magnetic dipoles revisited. Geophys Res Lett 25:2003-2006

Dyment J, Choi Y, Hamoudi M, Lesur V, Thébault E (2015) Global equivalent magnetization of the oceanic lithosphere. Earth Planet Sci Lett 430:54-65. doi:10.1016/j.epsl.2015.08.002

Hamoudi M, Thebault E, Lesur V, Mandea M (2007) GeoForschungsZentrum Anomaly Magnetic MAp (GAMMA): a candidate model for the World Digital Magnetic Anomaly Map. Geochem Geophys Geosys 8 Q06023:10-10292007001638

Korhonen J, Fairhead JD, Hamoudi M, Hemant K, Lesur V, Mandea M, Maus S, Purucker M, Ravat D, Sazonova T, Thébault E (2007) Magnetic anomaly map of the world — carte des anomalies magnétiques du monde. Commission for Geological Map of the World 1st Edition. Paris, France

Lesur V, Rother M, Vervelidou F, Hamoudi M, Thébault E (2013) Post-processing scheme for modeling the lithospheric magnetic field. Solid Earth 4:105-118. doi:10.5194/sed-4-105-2013

Maus S, Sazonova T, Hemant K, Fairhead JD, Ravat D (2007) National geophysical data center candidate for the World Digital Magnetic Anomaly Map. Geochem Geophys Geosyst 8(Q06017). doi:10.1029/2007GC001643

Maus S, Barckhausen U, Berkenbosch H, Bournas N, Brozena J, Childers V, Dostaler F, Fairhead JD, Finn C, von Frese RRB, Gaina C, Golynsky S, Kucks R, Lühr H, Milligan P, Mogren S, Müller RD, Olesen O, Pilkington M, Saltus R, Schreckenberger B, Thébault E, Caratori Tontini F (2009) Emag2: a 2-arc min resolution earth magnetic anomaly grid compiled from satellite, airborne, and marine magnetic measurements. Geochem Geophys Geosyst 10(8). doi:10.1029/2009GC002471

Maus S (2010) An ellipsoidal harmonic representation of earth's lithospheric magnetic field to degree and order 720. Geochem Geophys Geosyst 11(Q06015). doi:10.1029/2010GC003026

Müller RD, Roest WR, Royer JY, Gahagan LM, Sclater JG (1997) Digital isochrons of the world's ocean floor. J Geophy Res 102:3211-3214

Müller RD, Sdrolias M, Gaina C, Roest WR (2008) Age, spreading rates and spreading asymmetry of the world's ocean crust. Geochem Geophys Geosyst 9(Q04006). doi:10.1029/2007GC001743

Milligan PR, Franklin R, Minty BRS, Richardson LM, Percival PJ (2010) Magnetic anomaly map of Australia (Fifth Edition). 1:15000 000 scale, Geoscience Australia, Canberra. doi:http://dx.doi.org/10.4225/25/5625903503F9A

Nakanishi M, Tamaki K, Kobayashi K (1989) Mesozoic magnetic anomaly lineations and seafloor spreading history of the northwestern Pacific J Geophys Res 94(B11). doi:10.1029/JB094iB11p15437

Nakanishi M, Tamaki K, Kobayashi K (1992) Magnetic anomaly lineations from Late Jurassic to Early Cretaceous in the west-central Pacific Ocean. Geophys J Int 109(3):701-719. doi:10.1111/j.1365-246X.1992.tb00126.x

Nakanishi M, Winterer EL (1998) Tectonic history of the Pacific-Farallon-Phoenix triple junction from Late Jurassic to Early Cretaceous: an abandoned spreading ridge in the central Pacific basin. J Geophys Res 103(B6):12453-12468. doi:10.1029/98JB00754

Patriat P (1987) Reconstitution de l'évolution du système de dorsales de I'ocean indien par les méthodes de la cinématique des plaques. Territoire des Terres Australes et Antarctique Francaises, Paris, pp 308

Quesnel Y, Catalàn M, Ishihara T (2009) A new global marine magnetic anomaly data set. J Geophys Res 114(B04106). doi:10.1029/2008JB006144

Ravat D, Finn C, Hill P, Kucks R, Phillips J, Blakely R, Bouligand C, Sabaka T, Elshayat A, Aref A, Elawadi E (2009) A preliminary, full spectrum, magnetic anomaly grid of the United States with improved long wavelengths for studying continental dynamics-A website for distribution of data: U.S. Geological Survey Open-File Report 2009-1258, p. 2. http://pubs.usgs.gov/ of/2009/1258/downloads/OF09-1258.pdf

Royer JY, Müller RD, Gahagan LM, Lawyer LA, Mayes CL, Nuernberg D, Sclater JG (1992) A global isochron chart. Tech. Rep. 117, 38Inst. for Geophys., Univ. of Texas, Austin

Sabaka TJ, Olsen N, Purucker ME (2004) Extending comprehensive models of the Earth's magnetic field with Ørsted and CHAMP data. Geophys J Int 159:521-547. doi:10.1111/j.1365-246X.2004.02421.x

Thébault E (2008) A proposal for regional modelling at the earth's surface, r-scha2d. Geophys J Int. doi:10.1111/j.1365-246X.2008.03823.x

Vervelidou F, Thébault E (2015) Global maps of the magnetic thickness and magnetization of the earth's lithosphere. Earth Planets Space 67(1):1-19. doi:10.1186/s40623-015-0329-5

Wonik T, Galdéano A, Hahn A, Mouge P (1992) Magnetic anomalies. In: Freeman R, St. Mueller S (eds). A Continent Revealed - the European Geotraverse. Atlas of Compiled Data. Cambridge University Press, Cambridge. pp 31-34

\section{Submit your manuscript to a SpringerOpen ${ }^{\circ}$ journal and benefit from:}

- Convenient online submission

- Rigorous peer review

- Immediate publication on acceptance

- Open access: articles freely available online

- High visibility within the field

- Retaining the copyright to your article

Submit your next manuscript at springeropen.com 